\title{
An Automated System for the Detection and Diagnosis of Kidney Lesions in Children from Scintigraphy Images
}

\author{
Matilda Landgren ${ }^{1,2}$, Karl Sjöstrand ${ }^{1,3}$, Mattias Ohlsson ${ }^{1,4}$, Daniel Ståhl ${ }^{1,2}$, \\ Niels Christian Overgaard ${ }^{2}$, Kalle Åström ${ }^{2}$, Rune Sixt ${ }^{5}$, and Lars Edenbrandt ${ }^{1}$ \\ 1 EXINI Diagnostics AB, Lund, Sweden \\ \{karl.sjostrand, mattias.ohlsson, lars.edenbrandt\}@exini.com \\ ${ }^{2}$ Centre for Mathematical Sciences, Lund University, Lund, Sweden \\ $\{$ nco,kalle\}@maths.lth.se \\ 3 Department of Informatics and Mathematical Modelling, \\ Technical University of Denmark, Kgs. Lyngby, Denmark \\ 4 Department of Theoretical Physics, Lund University, Lund, Sweden \\ 5 Queen Silvia Children's Hospital, Göteborg, Sweden
}

\begin{abstract}
Designing a system for computer aided diagnosis is a complex procedure requiring an understanding of the biology of the disease, insight into hospital workflow and awareness of available technical solutions. This paper aims to show that a valuable system can be designed for diagnosing kidney lesions in children and adolescents from ${ }^{99 m} \mathrm{Tc}$ DMSA scintigraphy images. We present the chain of analysis and provide a discussion of its performance. On a per-lesion basis, the classification reached an ROC-curve area of 0.96 (sensitivity/specificity e.g. 97\%/85\%) measured using an independent test group consisting of 56 patients with 730 candidate lesions. We conclude that the presented system for diagnostic support has the potential of increasing the quality of care regarding this type of examination.
\end{abstract}

Keywords: Computer Aided Diagnosis, Nuclear Imaging, Active Shape Models, Artificial Neural Networks.

\section{Introduction}

Proper medical treatment begins with a correct diagnosis. Medical imaging systems provide a wealth of information which provide possibilities as well as challenges for the interpreting physician. The processing of this information is a complex procedure, where collective knowledge in the field, familiarity with the specific examination procedure and technical equipment, patient history, and common sense come together in the formation of a diagnosis. Creating a fully automated system for processing information of this diversity is difficult; however there are situations where a computerized system can provide valuable diagnostic support. Computers excel at keeping track of large amounts of data and at

A. Heyden and F. Kahl (Eds.): SCIA 2011, LNCS 6688, pp. 489 500, 2011.

(C) Springer-Verlag Berlin Heidelberg 2011 
performing time-consuming and tedious tasks quickly. The combination of a human interpreter and a computerized system can therefore improve diagnostic accuracy [1. The main contribution of such a system is to improve sensitivity, i.e. avoiding oversight. This paper presents a fully automated system for detecting and diagnosing kidney lesions from 2D scintigraphy images. There are two main contributions of this system. First, it eliminates time-consuming manual procedures in currently used systems such as the delineation of the kidneys. Second, it provides objective diagnostic support on a per-lesion basis to physicians with limited experience with this type of examination.

\subsection{Clinical Background}

One of the most common bacterial infections among children is urinary tract infection, caused by bacterial growth. This condition may develop into pyelonephriti: 1 which, left untreated, may cause scars in the parenchyma of the kidneys (cf. Figure 1(a) for anatomical terms). Among the possible consequences of such lesions are future renal hypertension (high blood pressure) and renal failure [2]. Children with recurring infections are investigated for possible kidney lesions using an imaging method where a harmless agent known as dimercaptosuccinic acid (DMSA) is injected into the blood. DMSA accumulates in the kidneys and the local accumulation is proportional to the density of functional kidney cells 344 . Low accumulation is therefore indicative of locally reduced kidney function. To make it possible to image the amount of accumulation, DMSA is combined with a radioactive molecule, ${ }^{99 m} \mathrm{Tc}$, a weak gamma radiation emitter. A planar gamma detector is used to measure the amount of radiation emitted from the kidneys, thus forming an image of the renal function. The distribution of ${ }^{99 m} \mathrm{Tc}-\mathrm{DMSA}$ in the kidneys are normally homogeneous. When a patient has had repeated urinary tract infections with lowered kidney function as a result, this can be seen as wedge-shaped areas of locally reduced intensity in the scintigraphy image [4], cf. Figure 1(b).

\subsection{Related Work}

A segmentation method, specific to renal scintigraphy, is proposed in [5]. An automatic thresholding algorithm is used to segment each kidney. To avoid undersegmentation, pixels in an area around the initial boundary are classified as kidney pixels or background. This method does, however, not consider the fact that diseased kidneys may show lower uptake in wedge-shaped areas around its boundary. The paper also proposes a system for diagnosing the entire kidney as normal or abnormal based on a boundary curvature measure. The sensitivity and specificity of this method was $88 \%$ and $96 \%$ respectively.

A commercial ${ }^{99 m}$ Tc-DMSA analysis program is available from Hermes Medical Solutions [6]. This software presents various kidney-specific measurements and presents a statistical map of uptake deviations.

\footnotetext{
${ }^{1}$ Inflammation in the renal pelvis.
} 


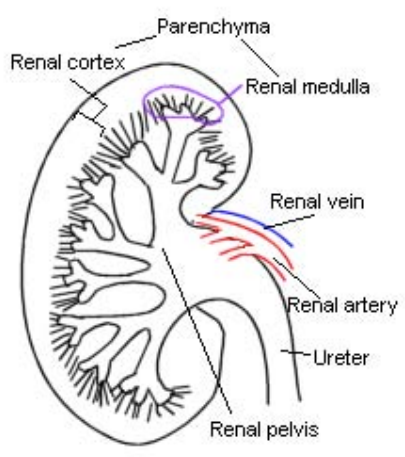

(a) A schematic image of the basic anatomy of the kidney.

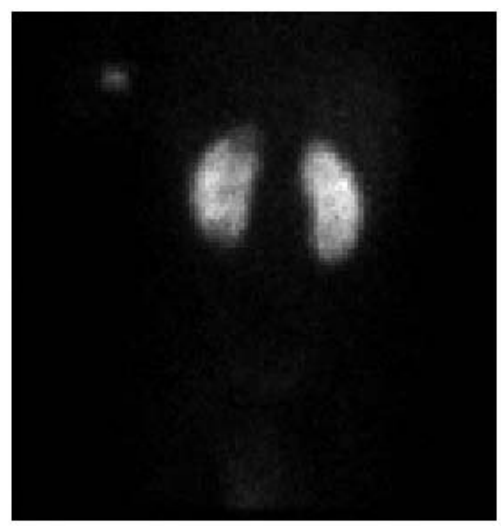

(b) A renal scintigraphy image where lesions are present in the upper and lower part of the left kidney, and the right kidney is normal. The image size is $128 \times 128$ pixels and the side of a pixel is $2.26 \mathrm{~mm}$.

Fig. 1 .

\section{Methods}

An overview of our system can be seen in Figure 2 and the different steps are explained in more detail below.

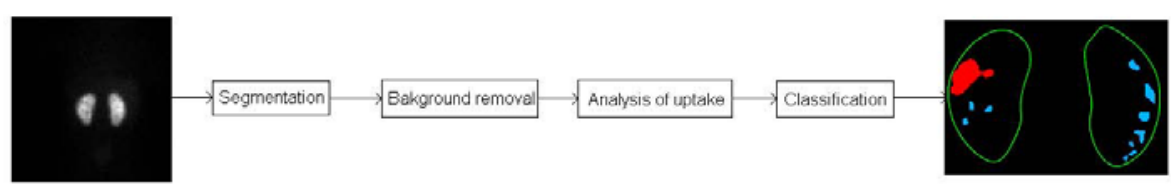

Fig. 2. An overview of the system components, from input image to a lesion-based classification

\subsection{Kidney Segmentation}

To segment and classify lesions we require some knowledge of relevant kidney anatomy; for instance we wish to build a map of normal ${ }^{99 m}$ Tc-DMSA uptake and to measure the size of a lesion relative to the entire kidney. As is evident from Figure 1(b), delineating the outer borders of the kidneys is a relatively easy task, the kidneys are mostly of high intensity while the background is considerably darker. However, lesions commonly distribute along the border, creating wedge-like regions of low intensity. To recover a plausible kidney border in such 
areas, strong prior information on the kidney shape must be incorporated. We believe that Active Shape Models (ASMs) [7] are a suitable approach for this purpose. An ASM models the distribution of landmarks along the boundary of a structure as a multivariate Gaussian distribution by means of a principal component analysis on the concatenated $x$ - and $y$-coordinates of all shapes. Tuning the parameters of this model to fit an object in a given image is carried out in an alternating fashion. First, each landmark of the model is moved to a position in its vicinity which most likely represents the object border. Then, the landmark configuration is relaxed using information from the statistical landmark model to ensure that the resulting shape is anatomically plausible.

To create the statistical model of kidney anatomy, a training set of 40 kidneys were annotated with 14 landmarks along the boundary. The model is of a right kidney; left kidneys were mirrored to be incorporated in the model. By mirroring the model, we obtain a segmentation tool which can be used on both kidneys under the assumption of no consistent difference between left and right kidneys [5]. The resulting shape model uses 10 principal axes which captures $95 \%$ of the training set variation.

Successful delineation of a kidney using the ASM scheme requires an initial estimate of the segmentation which is reasonably close to the actual structure. A bounding box containing the kidney to segment is easily obtained from the marginal image histograms. The position, size and rotation of this kidney is then estimated by computing the centroid and principal axes for the coordinates corresponding to pixels above a foreground intensity threshold. Using this information, the mean shape is translated such that its centroid coincides with the estimated kidney centroid. The shape is then rotated according to the principal axes. Finally, the shape is scaled in the directions of the principal axes according to the variance explained along each axis. Results of this initialization can be seen in the upper row of Figure 3 .

The ASM search for an improved fit from the initial segmentation guess is based on image edge information. Applying an edge detection algorithm on the raw image data is unwise; scintigraphy images exhibit uneven intensities due to the underlying Poisson process of nuclear decay. Neighboring pixels representing the same tissue type may display vastly different intensity levels just by chance. To reduce this effect while preserving more global edge structures we apply a bilateral image filter [8. Bilateral filters are similar to standard Gaussian blurring with the modification that the Gaussian bell is weighted in each position by the photometric distance between the central pixel and its neighbors. This has the effect of smoothing homogenous regions while preserving structure. As a final preparatory image modification, we encourage the ASM to disregard edges enclosed in bright areas to some extent by taking the square root of all intensity values, thus focusing more on the background/foreground edges of interest.

When moving a landmark to a new position, we search among 30 samples along profiles perpendicular to the shape as suggested in [7]. From these candidate positions, we select the one which maximizes the difference between the mean of inside samples and the mean of outside samples. An advantage of this 

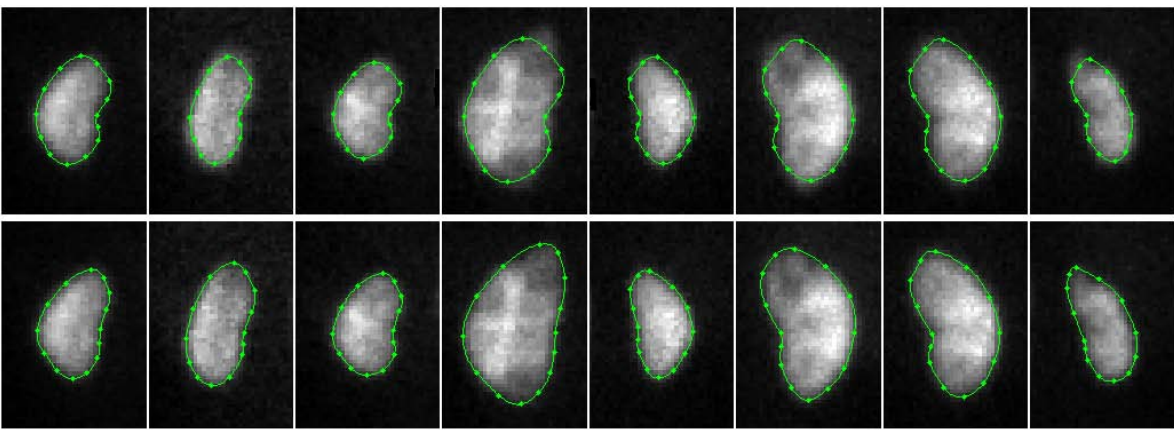

Fig. 3. Example of segmentations with the initialization in the upper row and the final segmentation in the lower row. In the fourth example from the left it can be seen that the segmentation algorithm recovers the low-intensity upper and lower kidney poles.

formulation is that it considers edges with background on the outside and foreground on the inside of the shape model, rather than any image edges. The algorithm is run until the landmark difference between iterations is sufficiently low. The second row of Figure 3 shows examples of resulting segmentations.

\subsection{Boundary Representation}

As stated above, the kidney shape model consists of 14 landmarks placed along the kidney image boundary. This results in a rather course representation of the outline. However, a larger number of landmarks is difficult to achieve since the kidney exhibits few distinctive anatomical points of reference as projected in ${ }^{99 m}$ Tc-DMSA images. Instead, we rely on a suitable interpolation technique to connect the landmarks accurately. The boundary is represented by a simple closed curve, making the use of Fourier descriptors [9] suitable. This boundary representation uses a combination of the discrete Fourier transform and Fourier series to calculate the Fourier coefficients $c_{k}$ and to recover a continuous boundary curve representation $f(t)$ respectively,

$$
c_{k}=\frac{1}{n} \sum_{j=0}^{n-1} e^{-2 \pi i j k / n} f_{j}, \quad f(t)=\sum_{k=-n / 2}^{n / 2} c_{k} e^{i k t} .
$$

Here $n=14$ is the number of landmarks, $f_{j}=x_{j}+i y_{j}, j=0 \ldots n-1$ represents the set of input landmarks, and $i$ is the complex unit. The complex boundary function $f(t)$, here sampled at 200 points, is a sum of harmonics of differing phase and frequency which leads to a globally smooth boundary suitable for describing the kidney boundary. Further, there exists convenient analytical expressions for the area and the centroid of the resulting shape 9 . The outlines in Figure 3 are interpolated using this technique; note how it better handles the curvedness of the outline than line segments would. 


\subsection{Background Removal}

The background radiation present in the ${ }^{99 m}$ Tc-DMSA kidney images is due to partial uptake in the blood and other organs. This also occurs behind and in front of the kidneys, as viewed from the gamma detector. This effect can be substantial and must be taken into account for accurate estimation of the kidney-specific ${ }^{99 m}$ Tc-DMSA uptake. Our approach is to create a smooth surface representing how the background radiation varies over the kidney, based on background samples outside, but close to, the kidney. We then subtract the intensities implied by this surface from the kidney area and a result of this can be seen in the top row of Figure 5 .

To represent a smoothly varying surface without sudden kinks or excessive bending, we use a smoothing thin-plate spline [10]. To obtain a smooth estimate of the background uptake, and avoid fitting the surface to noise, we choose to regularize the thin-plate spline rather than smoothing the image background samples. The resulting surface solves

$$
\arg \min _{f} \sum_{i=1}^{n}\left(z_{i}-f\left(x_{i}, y_{i}\right)\right)^{2}+\lambda \iint\left[\left(\frac{\partial^{2} f}{\partial x^{2}}\right)^{2}+2\left(\frac{\partial^{2} f}{\partial x \partial y}\right)^{2}+\left(\frac{\partial^{2} f}{\partial y^{2}}\right)^{2}\right] \mathrm{d} x \mathrm{~d} y
$$

where $\{z\}$ is the set of background samples outside the kidney, $\{x, y\}$ is the set of image coordinates of these samples, $f(x, y)$ is the thin-plate spline approximant and $\lambda \in[0, \infty)$ determines the stiffness of the plate. There exists a closed-form solution for solving this problem via a linear system of equations [10].

\subsection{Candidate Lesion Segmentation}

The most distinctive image feature of kidney lesions is reduced uptake of ${ }^{99 m} \mathrm{Tc}-$ DMSA. We therefore base our lesion segmentation approach on a pixel-wise statistical map of uptake in healthy kidneys and classify areas of a kidney as candidate lesions which exhibit significantly lowered uptake, measured on the $5 \%$ level. In order to create a map of normal uptake, we created a database of normal kidneys; six patients where both kidneys are considered normal, eleven patients with normal left kidneys, and 17 patients with normal right kidneys a total of 40 samples. Since kidneys have different shapes and sizes they must be transformed to a common frame of reference where we obtain an approximate pixel-wise anatomical correspondence. We use thin-plate spline interpolation of the 14 corresponding outline landmarks to this end where each warp is represented by a pair of thin-plate splines taking care of landmark deflections in the $x$ and $y$ directions respectively.

Besides this spatial normalization the images also require photometric normalization by an unknown multiplicative factor. This is a general challenge in many investigations in nuclear medicine as absolute uptake depends on many unknown biological and technical parameters. Our normalization approach matches image $A$ to image $B$ by multiplying $A$ by a factor such that the median of regions of $A$ with high intensities (almost certainly healthy) matches the median of the 


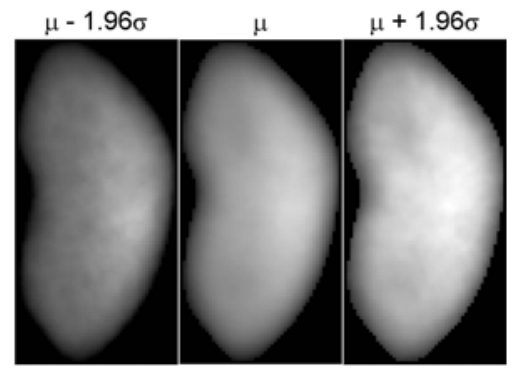

Fig. 4. The middle image shows the mean uptake of ${ }^{99 m}$ Tc-DMSA and the two outer images shows the uptake at the borders of the confidence interval

corresponding regions in $B$. In the normal database, all samples are normalized with respect to an arbitrarily chosen normal sample.

Empirical experience shows that a normal distribution is sufficiently accurate to describe the distribution of intensities. The parameters of these pixel-wise distributions are estimated by computing the mean and standard deviation of all normalized normal samples. Figure 4 shows the mean uptake and its $95 \%$ confidence interval. The middle row of Figure 5] shows the resulting statistical maps of $z$-values where tones of yellow towards red indicate regions lower than -2 standard deviations $(-2 z)$.

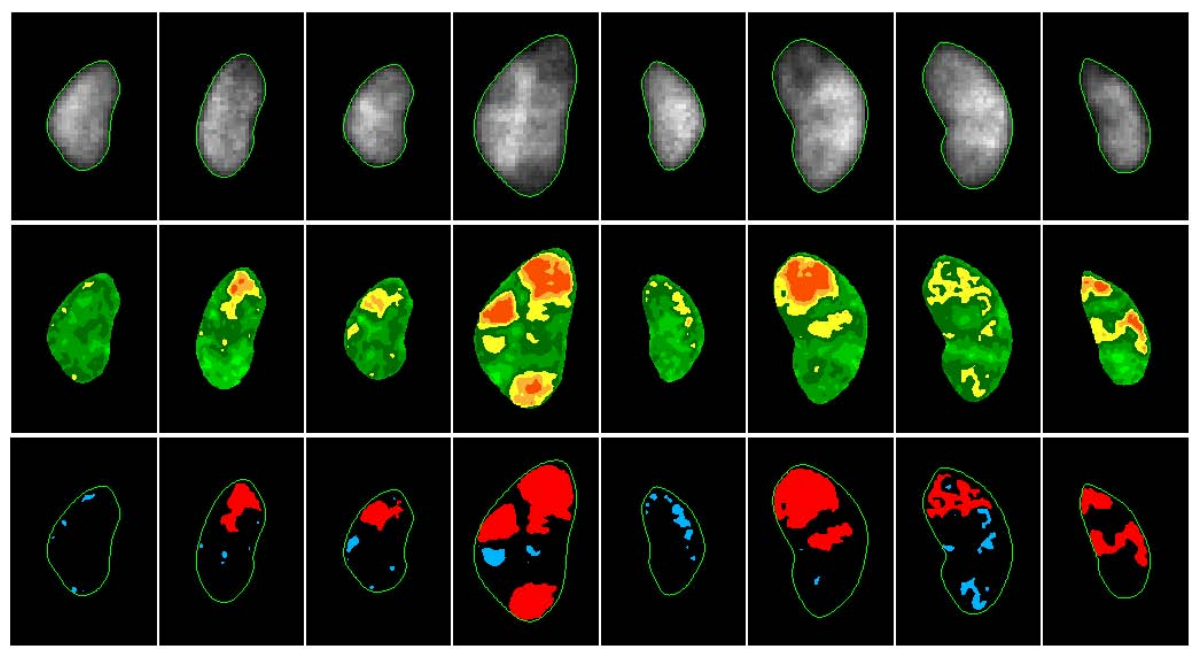

Fig. 5. Top row shows the resulting intensities when the background radiation has been subtracted. In the middle row a statistical maps of $z$-values for the selection of samples can be seen. Yellow towards red indicate areas lower than $-2 z$. Bottom row shows example of classification with LDA, the red areas are classified as scars and the blue ones are classified as healthy. 


\subsection{Classification}

The essence of a system for computer aided diagnosis (CAD) is a classification into normal or abnormal, or gradings thereof, either on a per-lesion basis or regarding the patient as a whole. Here, we classify each lesion as either normal (blue) or abnormal (red). Typically, such systems are tuned such that very few actual lesions are classified as normal (high sensitivity). This gives the interpreting physician the possibility to focus on lesion candidates classified as abnormal, thus streamlining the work and reducing the risk of oversight.

We conducted experiments with three classifiers, two relatively simple baseline classifiers and one state-of-the-art approach. For baseline experiments, we used linear and quadratic discriminant analysis (LDA, QDA), and the more advanced approach is represented by an artificial neural network (ANN). A set of features is calculated from each potential lesion in order to perform the classification. These relate both to the lesion shape and texture, as well as to the kidney and patient as a whole. The following set of features were used here:

Lesion edge closeness. This feature measures the distance from the lesion to the kidney edge. For each pixel position along the contour of the lesion, the distance to the closest point on the continuous kidney perimeter is found using numerical optimization. The smallest such distance, measured in millimeters, is returned.

Lesion major and minor axis length. Length in millimeters of the principal axes of the ellipse with the same second moments as the lesion.

Relative lesion area. The area of the lesion divided by the kidney area.

Lesion sum of z-scores. The sum of the $\mathrm{z}$-values within a lesion. The $\mathrm{z}$-values are obtained by subtracting the lesion normal database mean image and dividing by the database standard deviation image, making each lesion pixel $\mathcal{N}(0,1)$-distributed.

Lesion relative sum of z-scores. The sum of lesion z-values divided by the area of the lesion. Measured in z-scores per square millimeter.

Lesion localization. These two features measure the position of the lesion centroid relative to its bounding box in the $\mathrm{x}$ - and $\mathrm{y}$-directions. Since the scars are often located at the lateral wall of the kidney this measure can be an important addition to lesion edge closeness. Measures range from 0 to 1 with 1 corresponding to the most lateral/caudal positions and 0 corresponding to the most medial/cranial positions.

Lesion eccentricity. This feature measures the elongation of the lesion as the eccentricity of the ellipse with the same second moments as the lesion. Values range from 0 to 1 , where 0 represents a circle and 1 represents a line segment.

Lesion rate of extreme database deviation. This feature measures the proportion of the lesion which falls under 4 standard deviations when compared to the normal database.

Kidney separate function. This important measure quantifies the functional relation between the two kidneys of a patient. With equally functioning kidneys, this measure is at $50 \%$. Lower numbers indicate loss of kidney function. 
Kidney length. This feature is of importance since partially damaged kidneys are likely to suffer from impaired growth. Measured in millimeters.

Kidney area. Another way of measuring kidney growth. Measured in square millimeters.

Patient age. Age can be an important factor to control for as kidneys develop much during childhood.

To train the classifiers we created a training set consisting of 36 patients with a total of 483 candidate lesions. These lesions were classified as normal or abnormal by a leading specialist on interpreting ${ }^{99 m}$ Tc-DMSA renal scintigraphy images. The prevalence of lesions was $12 \%$. Separate from this training set, we created a test set from 56 patients with a total of 730 candidate lesions. The prevalence in the test set was $8 \%$.

Under the assumption that the vector of features for a patient follows a multidimensional Gaussian distribution, LDA and QDA provide optimal classification in terms of minimizing error rate [1]. Further, LDA assumes that normal and abnormal candidate features share the same covariance matrix while QDA allow for different such matrices. LDA and QDA assign a candidate lesion to the class $k$ that maximizes

$$
\begin{gathered}
\delta_{k}=x^{T} \boldsymbol{\Sigma}^{-1} \mu_{k}-\frac{1}{2} \mu_{k}^{T} \boldsymbol{\Sigma}^{-1} \mu_{k}+\log \pi_{k}, \quad \text { and } \\
\delta_{k}=-\frac{1}{2} \log \left|\boldsymbol{\Sigma}_{k}\right|-\frac{1}{2}\left(x-\mu_{k}\right)^{T} \boldsymbol{\Sigma}_{k}^{-1}\left(x-\mu_{k}\right)+\log \pi_{k}
\end{gathered}
$$

respectively, where $k \in\{$ normal, abnormal $\}, x$ represents the input feature vector to test for, $\boldsymbol{\Sigma}$ represents a covariance matrix, $\mu_{k}$ is the mean feature vector for class $k$, and $\pi_{k}$ is the prior probability for class $k$.

The ANN classification model was implemented as an ensemble of multilayer perceptrons (MLP), where each MLP consisted of one hidden layer (7 nodes). Training was carried out by minimizing a cross-entropy error with an additional weight elimination term [12 to allow for a possible regularization of the ensemble. Four-fold cross validation was used during the model selection phase and the final ensemble model used on the test set was created using 3fold cross splitting, repeated 10 times, resulting in an ensemble of size 30 . The average output of the MLPs was used as the ensemble prediction.

Examples of lesions classified by LDA can be seen in the bottom row of Figure 5 .

\section{$3 \quad$ Results}

The segmentation works well both on kidneys with normal ${ }^{99 m}$ Tc-DMSA uptake and on kidneys with lower uptake, cf. Figure 3. The shape model also makes sure that the shape of a kidney is maintained. The segmentation has been evaluated on 40 kidneys and the rate of acceptable segmentations is around $95 \%$. Most of the unacceptable segmentations have minor errors; most common is an unsatisfying segmentation of the upper and lower pole of the kidney. 
Table 1. Classification results as measured on the test set of 730 candidate lesions

\begin{tabular}{|l|c|c|c|}
\hline & LDA & QDA & ANN \\
\hline Area under ROC curve (AUC) & 0.964 & 0.935 & 0.960 \\
Sensitivity (\%) & 96.5 & 96.5 & 96.5 \\
Specificity (\%) & 84.8 & 61.2 & 83.4 \\
Positive Predictive value (\%) & 35.0 & 17.4 & 32.9 \\
Negative Predictive value (\%) & 99.7 & 99.5 & 99.6 \\
Mis-classification rate (\%) & 14.2 & 36.0 & 15.6 \\
\hline
\end{tabular}

The lesion segmentation algorithm based on the database of normal uptake detected $100 \%$ of diagnosed lesions in the training and test data sets. We did not have the opportunity to assess the accuracy of candidate lesion geometry.

The classification was validated on a test set of 730 possible lesions and the performance of the different classifiers can be seen in Table 11. We fixed the sensitivity at a high value, here $96.5 \%$, since this lessens the risk of classifying an actual lesion as normal while maintaining reasonable specificity. LDA and ANN show similar performance with high specificity and negative predictive value. QDA has notably lower specificity and positive predictive value as well as a larger misclassification rate. Receiver Operating Characteristic (ROC) curves for each classifier can be seen in Figure 6.

The program has been developed using MATLAB. The computation time for the analysis of one patient ranged from 5-9 seconds when running on a $2.2 \mathrm{GHz}$ Windows PC with $1.5 \mathrm{Mb}$ of RAM.

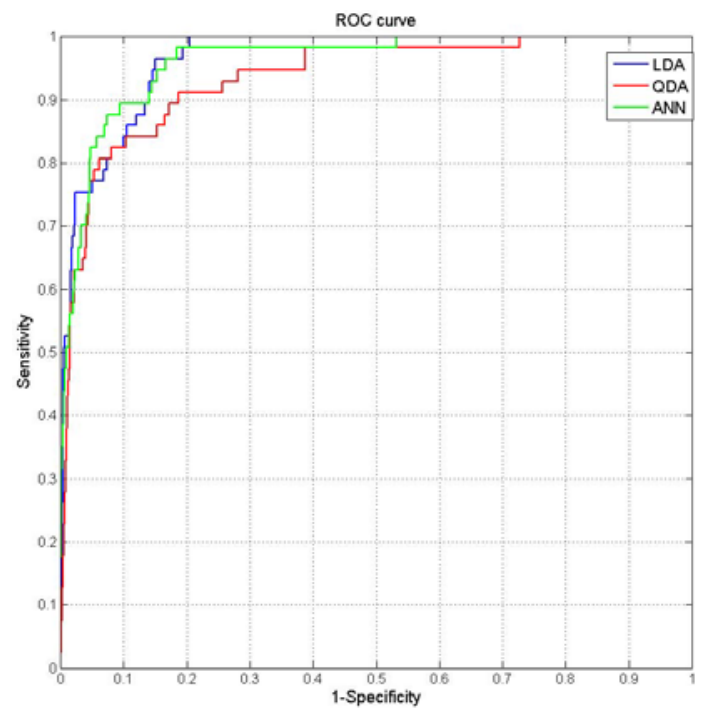

Fig. 6. ROC curves for LDA (blue), QDA (red), and ANN (green). LDA and ANN perform similarly while QDA does slightly worse. 


\section{Discussion}

We have shown that an accurate system for the diagnosis of kidney lesions can be created. Similar systems have previously been shown to increase diagnostic accuracy in practice, particularly by increasing sensitivity [1. This means that physicians are less likely to miss non-conspicuous lesions. In contrary to many presently used systems, our approach is fully automatic and quick. This has the potential of increasing care effectiveness and relieving the interpretation from some of its inherent subjectiveness.

In our classification experiments, linear discriminant analysis performed at least as good as the more flexible alternatives. Cross-validation results for the artificial neural network approach was significantly better than those of LDA and QDA, but performance dropped when measured on the independent test set. LDA did not suffer from this effect. This is most likely due to the rigid nature of LDA, rather than that the assumptions of LDA are fulfilled by the data 11. It also seems as if the classification problem is rather linear in nature, in fact, one can obtain a reasonably good system (95\% AUC) using relative lesion area as a single feature. Fair comparisons with CAD systems for other diagnoses are difficult, but it is interesting to note that positive predictive values of typical mammography systems are $2-3 \%$ [13. Here, we reach $35 \%$.

The lesion detection system detected 730 lesions in the test material $-8 \%$ of these represented actual lesions. This stresses the importance of offering classification of detected findings. The work involved in classifying 730 findings from scratch is far greater than reviewing around 60 candidates classified as abnormal paired with a quick review of remaining lesions.

In this paper, we have used data from a single hospital. In future work we will evaluate the method on material from more centers and a bigger variety of cameras. Further, we wish to develop the user interface to better fit typical hospital workflow, including integration with image storage and retrieval systems, and to provide a system for (semi-)automatic reporting. One may also consider providing a diagnosis for the patient as a whole, based on the diagnoses of the individual lesions, in order to quantify the risk of future renal malfunction.

\section{References}

1. Sadik, M., Suurkula, M., Höglund, P., Järund, A., Edenbrandt, L.: Improved classifications of planar whole-body bone scans using a computer-assisted diagnosis system: a multicenter, multiple-reader, multiple-case study. J. Nucl. Med. 50(3), 368-375 (2009)

2. Fotter, R.: Pediatric Uroradiology. Springer, Heidelberg (2008)

3. Piepsz, A., Colarinha, P., Gordon, I., Hahn, K., Olivier, P., Roca, I., Sixt, R., van Velzen, J.: Guidelines on $99^{m}$ Tc-DMSA scintigraphy in children. Eur. J. Nucl. Med. 28(3), 37-41 (2001)

4. Jonson, B., Wollmer, P.: Klinisk Fysiologi. Liber AB (2005) (in swedish)

5. Marcuzzo, M., Masiero, P.R., Scharcanski, J.: Quantitative Parameters for the Assessment of Renal Scintigraphic Images. In: 29th Annual International Conference of the IEEE Engineering in Medicine and Biology Society, pp. 3438-3441 (2007) 
6. Hermes Medical Solutions, http://www .hermesmedical . com/index . lasso?id=128

7. Cootes, T.F., Taylor, C.J., Cooper, D.H., Graham, J.: Active Shape Models - their training and application. Computer Vision and Image Understanding 61(1), 38-59 (1995)

8. Tomasi, C., Manduchi, R.: Bilateral Filtering for Gray and Color Images. In: Proceedings of the 1998 IEEE International Conference on Computer Vision, India (1998)

9. Sjostrand, K., Ericsson, A., Larsen, R.: On the Alignment of Shapes Represented by Fourier Descriptors. In: SPIE International Symposium on Medical Imaging, USA (2006)

10. Bookstein, F.L.: Principal Warps: Thin-Plate Splines and the Decomposition of Deformations. IEEE Transactions on Pattern Analysis and Machine Intelligence 11(6), $567-585(1989)$

11. Hastie, T., Tibshirani, R., Friedman, J.: The Elements of Statistical Learning. Springer, Heidelberg (2008)

12. Hanson, S.J., Pratt, L.Y.: Comparing biases for minimal network construction with back-propagation. In: Touretzky, D.S. (ed.) Advances in Neural Information Processing Systems, pp. 177-185 (1989)

13. Freer, T.W., Ulissey, M.J.: Screening Mammography with Computer-aided Detection: Prospective Study of 12,860 Patients in a Community Breast Center. Radiology 220, 781-786 (2001) 